\title{
Circulating EZH2-positive T cells are decreased in multiple sclerosis patients
}

Sunny Malhotra ${ }^{1 *}$, Luisa M. Villar², Carme Costa' ${ }^{2}$ Luciana Midaglia' ${ }^{1}$, Marta Cubedo ${ }^{3}$, Silvia Medina ${ }^{2}$, Nicolás Fissolo ${ }^{1}$, Jordi Río ${ }^{1}$, Joaquín Castilló ${ }^{1}$, José C. Álvarez-Cermeño ${ }^{2}$, Alex Sánchez ${ }^{4,5}$, Xavier Montalban ${ }^{1}$ and Manuel Comabella ${ }^{1 *}$

\begin{abstract}
Background: Recent studies in experimental autoimmune encephalomyelitis, an animal model of multiple sclerosis (MS), suggest an involvement of the histone methyltransferase enhancer of zeste 2 polycomb repressive complex 2 subunit (EZH2) in important processes such as cell adhesion and migration.

Methods: Here, we aimed to expand these initial observations by investigating the role of EZH2 in MS. mRNA expression levels for EZH2 were measured by real-time PCR in peripheral blood mononuclear cells (PBMC) from 121 MS patients (62 untreated and 59 receiving treatment) and 24 healthy controls.

Results: EZH2 expression levels were decreased in PBMC from untreated patients compared to that from controls, and treatment significantly upregulated EZH2 expression. Expression of miR-124 was increased in MS patients compared to controls. Blood immunophenotyping revealed EZH2 expression mostly restricted to CD4+ and CD8+ T cells, and circulating EZH2+ CD4+ and CD8+ T cells were decreased in untreated MS patients compared to controls. CD8+ T cells expressing EZH2 exhibited a predominant central memory phenotype, whereas EZH2+ CD4+ T cells were of effector memory nature, and both T cell subsets produced TNF-a. EZH2+ T cells were enriched in the cerebrospinal fluid compartment compared to blood and were found in chronic active lesions from MS patients. EZH2 inhibition and microarray analysis in PBMC was associated with significant downregulation of key T cell adhesion molecules.

Conclusion: These findings suggest a role of EZH2 in the migration of T cells in MS patients. The observation of TNF-a expression by $\mathrm{CD} 4+$ and $\mathrm{CD} 8+\mathrm{T}$ cells expressing EZH2 warrants additional studies to explore more in depth the pathogenic potential of EZH2+-positive cells in MS.
\end{abstract}

Keywords: Multiple sclerosis, EZH2, Treatment, Migration, Adhesion molecules

\section{Background}

Enhancer of zeste 2 polycomb repressive complex 2 subunit (EZH2) is a histone methyltransferase that serves as the catalytic subunit of the polycomb repressive complex 2 , a protein complex that regulates gene expression by methylating nucleosomal histone $\mathrm{H} 3$ at lysine 27 (H3K27) on the promoter of its target genes [1]. The identification of a cytosolic methyltransferase EZH2-containing complex suggested that, in addition to its role methylating histones, EZH2 could also be involved in the regulation of

\footnotetext{
* Correspondence: sunnymalhotra4u24@gmail.com;

manuel.comabella@vhir.org

${ }^{1}$ Servei de Neurologia-Neuroimmunologia, Centre d'Esclerosi Múltiple de Catalunya (Cemcat), Institut de Recerca Vall d'Hebron (VHIR), Hospital Universitari Vall d'Hebron, Universitat Autònoma de Barcelona, Barcelona, Spain

Full list of author information is available at the end of the article
}

extra-nuclear signaling pathways, in particular actin polymerization-dependent processes [2]. Recent investigation on its cytoplasmic role has revealed that EZH2 interacts with cytosolic proteins such as talin and the guanine nucleotide-exchange factor vav1 that link integrin molecules to the actin cytoskeleton, suggesting the potential implication of EZH2 in cell adhesion and migration processes [3]. Interestingly, mice lacking the EZH2 gene exhibited attenuated experimental autoimmune encephalomyelitis (EAE) disease progression due to the inability of EZH2-deficient cells, particularly neutrophils and dendritic cells, to reach the site of inflammation [3]. Taking into consideration the findings of EZH2 in EAE mice and its implication in important processes for the pathogenesis of multiple sclerosis

(c) The Author(s). 2018 Open Access This article is distributed under the terms of the Creative Commons Attribution 4.0 International License (http://creativecommons.org/licenses/by/4.0/), which permits unrestricted use, distribution, and reproduction in any medium, provided you give appropriate credit to the original author(s) and the source, provide a link to the Creative Commons license, and indicate if changes were made. The Creative Commons Public Domain Dedication waiver (http://creativecommons.org/publicdomain/zero/1.0/) applies to the data made available in this article, unless otherwise stated. 
such as cell adhesion and migration, we believe that EZH2 may also be playing a role in multiple sclerosis and contribute to the inflammatory component observed in the central nervous system (CNS) of patients. Hence, the purpose of the present study was to explore the role of EZH2 in the disease by measuring the gene expression levels of EZH2 and associated molecules in peripheral blood cells from untreated and treated multiple sclerosis patients and by characterizing the immune cell populations responsible for EZH2 expression.

\section{Methods}

\section{Patients}

Initial cohort

Messenger RNA (mRNA) expression levels of EZH2, talin 1 (TLN1), and VAV1 were determined in peripheral blood mononuclear cells (PBMC) from a first cohort of 24 healthy controls (HC) and 62 treatment-naïve multiple sclerosis patients. The case group included 25 patients with relapsing-remitting multiple sclerosis (RRMS), 20 patients with secondary progressive multiple sclerosis (SPMS), and 17 patients with primary progressive multiple sclerosis (PPMS). The RRMS group included 20 patients in clinical remission and 5 patients whose blood was drawn at the time of an acute relapse.

\section{Validation cohort}

In order to replicate EZH2 findings, mRNA expression levels for EZH2 were also measured in PBMC from an independent validation cohort comprised of $12 \mathrm{HC}$ and 13 treatment-naïve multiple sclerosis patients. Considering that EZH2 expression levels in the initial cohort were similar between different clinical forms of the disease, for the validation cohort, only patients with RRMS were included.

\section{Treated cohort}

EZH2 and TLN1 mRNA expression levels were determined in an additional cohort of 59 RRMS patients treated for at least 1 year with interferon-beta $(n=$ $17)$, glatiramer acetate $(n=15)$, fingolimod $(n=16)$, or natalizumab $(n=11)$. Expression levels for these genes were compared with those observed in a subgroup of 14 untreated RRMS patients included in the initial cohort.

The study was approved by the local Ethics Committee [EPA(AG)57/2013(3834)], and participants gave written informed consent. Tables 1 and 2 summarize demographic and baseline clinical characteristics of multiple sclerosis patients from the initial, validation, and treated cohorts and the HC included in the study.
Sample collection and determination of mRNA expression levels of EZH2, TLN1, and VAV1 by real-time PCR

$\mathrm{PBMC}$ from multiple sclerosis patients and $\mathrm{HC}$ were isolated by Ficoll-Isopaque density gradient centrifugation (Gibco BRL, Life Technologies LTD, UK) and stored in liquid nitrogen until used. Total RNA was extracted from PBMC using an RNeasy kit (Quiagen, Santa Clarita, USA) and cDNA synthesized using the High-Capacity cDNA Archive kit (Applied Biosystems, Foster City, CA, USA). mRNA expression levels for EZH2, TLN1, and VAV1 were determined with TaqMan ${ }^{\circ}$ probes specific for the gene (Applied Biosystems). The housekeeping gene glyceraldehyde3-phosphate dehydrogenase (GAPDH) was used as an endogenous control (Applied Biosystems). Assays were run on the ABI PRISM ${ }^{\circ} 7900 \mathrm{HT}^{2}$ system (Applied Biosystems), and data were analyzed with the $2^{-\Delta \Delta C \mathrm{~T}}$ method [4].

\section{Determination of microRNA expression levels by real-time PCR}

Expression levels for miR-124 and miR-155 were determined according to sample availability in PBMC from a subgroup of $18 \mathrm{HC}$ and 21 untreated multiple sclerosis patients (15 RRMS and 6 SPMS patients) who were also included in the initial cohort. Additional file 1: Table S1 summarizes demographic and main clinical characteristics of individuals included for this part of the study. PBMC were collected and processed in the same conditions as described in the previous section. Expression levels for miR-124 and miR-155 were measured with Taq $\mathrm{Man}^{\ominus}$ probes specific for the microRNAs (Applied Biosystems) using RNU $6 \mathrm{~b}$ as endogenous control. Analysis was performed as described above with the $2^{-\Delta \Delta C T}$ method [4].

\section{EZH2 immunophenotyping}

EZH2 protein expression was determined by flow cytometry according to sample availability in PBMC from $13 \mathrm{HC}$ [9 females (69.2\%); mean age (standard deviation), 35.8 years (10.9)] and 10 RRMS patients [5 females (50\%); mean age, 32.1 years (13.5); mean disease duration, 4.5 (3.5)] at baseline and after 1 year of natalizumab treatment. Only one MS patient and one $\mathrm{HC}$ were also included in the initial cohort whereas the remaining individuals corresponded to new multiple sclerosis patients and HC. EZH2 expression was also determined in cerebrospinal fluid (CSF) cells from 3 untreated RRMS patients [2 females (66.7\%); mean age, 34.3 years (11.9); mean disease duration, 0.4 (0.5)]. CSF samples were collected by lumbar puncture for clinical purposes and centrifuged at $1200 \mathrm{~g}$ for $15 \mathrm{~min}$. Supernatants were stored at $-80{ }^{\circ} \mathrm{C}$ until processed for clinical tests and CSF cells resuspended in PBS and labeled as described below. 
Table 1 Demographic and baseline clinical characteristics of the MS patients and healthy controls

\begin{tabular}{|c|c|c|c|c|c|}
\hline Baseline characteristics & $\mathrm{HC}$ & RRMS & SPMS & PPMS & Relapse \\
\hline \multicolumn{6}{|l|}{ Initial cohort } \\
\hline N & 24 & 20 & 20 & 17 & 5 \\
\hline Age (years) & $30.2(7.2)$ & $30.0(7.8)$ & $45.7(8.7)$ & $50.2(7.3)$ & $30.8(8.7)$ \\
\hline Female/male (\% women) & 18/6 (75.0) & $10 / 10(50.0)$ & $11 / 9(55.0)$ & $11 / 6(64.7)$ & $2 / 3(40.0)$ \\
\hline Duration of disease (years) & - & $4.8(4.6)$ & $11.5(7.6)$ & $12.2(7.9)$ & $2.2(2.7)$ \\
\hline EDSS $^{a}$ & - & $1.7(1.0-4.2)$ & $4.0(3.5-5.1)$ & $6.0(4.0-6.0)$ & $3.0(2.5-5.3)$ \\
\hline Numbers of relapses ${ }^{b}$ & - & $2.2(0.7)$ & $0.8(0.8)$ & - & $2.6(1.3)$ \\
\hline \multicolumn{6}{|l|}{ Validation cohort } \\
\hline N & 12 & 13 & & & \\
\hline Age (years) & $28.2(6.0)$ & $37.6(9.3)$ & & & \\
\hline Female/male (\% women) & $8 / 3(72.7)$ & $12 / 2(85.7)$ & & & \\
\hline Duration of disease (years) & - & $3.8(3.2)$ & & & \\
\hline $\mathrm{EDSS}^{\mathrm{a}}$ & - & $2.5(1.0-3.5)$ & & & \\
\hline Numbers of relapses ${ }^{b}$ & - & $2.5(0.8)$ & & & \\
\hline
\end{tabular}

\section{Monoclonal antibodies}

The following monoclonal antibodies were used in the study: EZH2-Alexa Fluor 488, CD197-PE (CCR7-PE), CD3-PE, granulocyte/macrophage colony-stimulating factor (GM-CSF)-PE, CD16-PE-Cy5, tumor necrosis factor (TNF)- $\alpha$-PercP-Cy5.5, CD19-PE-Cy7, CD45RO-APC, CD56-APC, CD8-APC-H7, CD14-APC-H7, CD3-BV421, CD45-V450, CD45-V500 (all from BD Biosciences, San Diego, CA), and IL-17-APC (R\&D Systems, Minneapolis, $\mathrm{MN})$.

\section{Characterization of EZH2 expression by CSF cells}

CSF cells were stained for $30 \mathrm{~min}$ at $4{ }^{\circ} \mathrm{C}$ in the dark with the appropriate amounts of monoclonal antibodies recognizing the surface antigens. Subsequently, cells were washed with PBS, fixed and permeabilized for $20 \mathrm{~min}$ at $4{ }^{\circ} \mathrm{C}$ in the dark with Cytofix/Cytoperm Kit (BD Biosciences), washed twice with Perm/Wash solution
(BD Biosciences) and stained intracellularly for $30 \mathrm{~min}$ at $4{ }^{\circ} \mathrm{C}$ in the dark with a monoclonal antibody recognizing EZH2, and washed and analyzed in a FACSCanto II flow cytometer (BD Biosciences).

\section{Intracellular cytokine staining}

Aliquots of $10^{6}$ PBMC were resuspended in $1 \mathrm{ml}$ of complete medium with $50 \mathrm{ng} / \mathrm{ml}$ phorbol 12-myristate 13-acetate (PMA) (Sigma-Aldrich, St. Louis, MO) and $750 \mathrm{ng} / \mathrm{ml}$ ionomycin (Sigma-Aldrich), in the presence of $2 \mu \mathrm{g} / \mathrm{ml}$ brefeldin A (GolgiPlug, BD Biosciences) and $2.1 \mu \mathrm{M}$ monensin (Golgi Stop, BD Biosciences) in polypropylene tubes, and incubated for $4 \mathrm{~h}$ at $37{ }^{\circ} \mathrm{C}$ in $5 \%$ $\mathrm{CO}_{2}$. Cells were washed in PBS and surface stained as indicated above. Afterward, cells were fixed and permeabilized for $20 \mathrm{~min}$ at $4{ }^{\circ} \mathrm{C}$ in the dark with Cytofix/ Cytoperm Kit (BD Biosciences), washed twice with Perm/Wash solution (BD Biosciences), and stained with

Table 2 Summary of demographic and baseline clinical characteristics of the treated MS cohort

\begin{tabular}{|c|c|c|c|c|c|}
\hline Characteristics & UNT & IFN & GA & $\mathrm{FG}$ & NTZ \\
\hline$N$ & 14 & 17 & 15 & 16 & 11 \\
\hline Age (years) & $28.3(6.3)$ & $34.8(7.5)$ & $32.3(7.9)$ & $30.3(7.8)$ & $27.7(14.5)$ \\
\hline Female/male (\% women) & $8 / 6(57.2)$ & $9 / 8(52.9)$ & 8/7 (53.3) & $11 / 5(68.7)$ & $7 / 4(63.6)$ \\
\hline Duration of disease (years) & $3.3(2.7)$ & $5.0(10.7)$ & $6.7(5.8)$ & $3.0(3.8)$ & $6.1(7.0)$ \\
\hline EDSS $^{a}$ & $1.8(1.4-2.5)$ & $1.6(1.0-2.0)$ & $2.2(1.5-3.0)$ & $1.6(1.0-2.0)$ & $2.5(1.6-3.5)$ \\
\hline Numbers of relapses ${ }^{b}$ & $2.0(0.8)$ & $1.5(0.8)$ & $2.3(1.5)$ & $2.2(0.7)$ & $1.9(0.6)$ \\
\hline
\end{tabular}

Data are expressed as mean (standard deviation) unless otherwise stated

UNT untreated relapsing-remitting MS patients, IFN interferon-beta, GA glatiramer acetate, FG fingolimod, NTZ natalizumab

${ }^{a}$ Data are expressed as mean (interquartile range) and refers to EDSS at the time of treatment onset

${ }^{\mathrm{b}}$ The number of relapses in the 2 years before treatment onset 
monoclonal antibodies recognizing GM-CSF, TNF- $\alpha$, and IL-17.

\section{Flow cytometry analysis}

Cells were always analyzed within $1 \mathrm{~h}$ of staining. Mean autofluorescence values were set using appropriate negative isotype controls. Data analysis was performed using FACSDiva Software V.8.0 (BD Biosciences). A gate including lymphocytes and monocytes and excluding debris and apoptotic cells was established; a minimum amount of 30,000 events for PBMC samples and 500 events for CSF cells were analyzed.

\section{EZH2 expression in EAE mice}

Anesthetized C57BL/6 mice were immunized by subcutaneous injections of PBS containing $50 \mu \mathrm{g}$ of $\mathrm{MOG}_{35-55}$ (Proteomics Section, Universitat Pompeu Fabra, Barcelona, Spain) or PBS, emulsified in complete Freund's adjuvant (Sigma Chemical, St. Louis, MO, USA), and supplemented with $2 \mathrm{mg} / \mathrm{ml}$ Mycobacterium tuberculosis H37RA (Difco Laboratories, Detroit, MI, USA). The animals received an additional intravenous injection of $150 \mathrm{ng}$ pertussis toxin in $100 \mu \mathrm{l}$ PBS on the day of immunization and again $48 \mathrm{~h}$ later. Four animals per group (EAE or controlsPBS) were sacrificed at $8,16,22,29,36$, and 50 days post-immunization, and spinal cord tissue was subsequently obtained. mRNA expression levels of EZH2 and CD3e were determined by real-time PCR as previously described. Changes in gene expression were always compared with animals treated with PBS at the respective days.

\section{EZH2 expression in human brain tissue \\ Samples}

Paraffin-embedded brain samples from RRMS patients and non-neurological controls were provided by the UK Multiple Sclerosis Tissue Bank. Tissue sections were stained with hematoxylin and eosin (HE) and Klüver-Barrera (KB) for inflammation and demyelination assessment. Ten samples from multiple sclerosis patients with chronic active lesions and four control samples were selected for the study (demographic and clinical information was not available for these patients).

\section{Immunohistochemistries}

Immunostainings were developed with the automated Benchmark XT platform from Ventana Medical System. Briefly, 4- $\mu \mathrm{m}$-thick, paraffin-embedded serial sections were deparaffinized with EZ prepTM (Ventana Medical System). Antigen retrieval was performed with Cell Conditioning $1 \mathrm{pH}=8$ (Ventana Medical System) for $30 \mathrm{~min}$. Endogenous peroxidase activity was blocked with hydrogen peroxide $3 \%$. Samples were incubated with rabbit anti-EZH2 (clone EPR9307(2), Abcam) for $36 \mathrm{~min}$ and visualized with ultraView Universal DAB (Ventana Medical
Systems). Subsequently, samples were kept at $95{ }^{\circ} \mathrm{C}$ for $8 \mathrm{~min}$ and incubated with rabbit anti-CD4 (clone SP35, Ventana Medical System) or rabbit anti-CD8 (clone SP57, Ventana Medical System) for $40 \mathrm{~min}$ and visualized with ultraView Universal Alkaline Phosphatase Red Detection (Ventana Medical Systems). All samples were counterstained with hematoxylin.

\section{Immunostaining assessment}

A range between 5 and 20 pictures were taken for each multiple sclerosis sample. Total CD4+ and CD8+ T cells and double-positive EZH2 and $\mathrm{CD} 4$ or $\mathrm{CD} 8$ cells were counted. The percentages of double-positive cells were calculated with respect to the total of CD4+ or CD8+ T cells.

\section{EZH2 blocking and gene expression microarrays}

PBMC from 7 untreated RRMS patients [5 females (71.4\%); mean age, 39.0 years (8.0); mean disease duration, 7.0 years (5.1)] were plated into 24-well plates for $24 \mathrm{~h}$ in the presence or absence of an EZH2 inhibitor (histone deacetylase inhibitor suberoylanilide hydroxamic acid-SAHA) at $1 \mu \mathrm{g} /$ $\mu \mathrm{l}$ concentration. After $24 \mathrm{~h}$, cells were harvested and total RNA isolated using the RNeasy kit (Quiagen) and hybridized to Affymetrix Human Transcriptome Arrays (HTA 2.0) (Affymetrix, Santa Clara, CA, USA) according to the manufacturer's protocol (GeneChip WT Pico Reagent Kit (Affymetrix)).

\section{Statistical analysis}

Statistical analysis was performed by using the SPSS 17.0 package (SPSS Inc., Chicago, IL) for MS Windows. Comparisons of mRNA expression levels for EZH2, TLN1, and VAV1; expression levels for miR-124 and miR-155; and the percentage of EZH2-positive cells between the different study groups were performed by parametric and non-parametric tests depending on the applicability conditions. Real-time PCR data were expressed as fold change in gene expression in controls relative to the whole group of multiple sclerosis patients and patients stratified according to the different clinical forms, in RRMS patients in clinical remission relative to patients in relapse, and in treated RRMS relative to untreated patients. For microarray analysis, images were processed with AGCC, Affymetrix GeneChip Command Console, to generate .CEL files. Raw expression values obtained directly from .CEL files were pre-processed using the RMA method [5]. These normalized values were the basis for all the subsequent analyses. Previous to any analysis data were submitted to non-specific filtering to remove low-signal genes (those genes whose mean signal in each group did not exceed a minimum threshold) and low-variability genes (those genes whose standard deviation between all samples did not exceed a minimum threshold). The selection of differentially expressed genes between the untreated and the EZH2 
blocking conditions was based on a linear model analysis with empirical Bayes moderation of the variance estimates following the methodology developed by Smyth [6]. In order to deal with the multiple testing issues derived from the fact that many tests (one per gene) were performed simultaneously, $p$ values were adjusted to obtain strong control over the false discovery rate using the Benjamini and Hochberg method [7].

\section{Results}

EZH2 expression is decreased in multiple sclerosis patients In order to investigate the role of EZH2 in multiple sclerosis, we first measured the mRNA expression levels of EZH2 and EZH2-associated genes in PBMC from an initial cohort of 62 untreated multiple sclerosis patients and $24 \mathrm{HC}$. As shown in Fig. 1a, expression levels for EZH2, TLN1, and VAV1 were significantly decreased in PBMC from the whole multiple sclerosis group compared to controls. Further stratification of the multiple sclerosis group into the different clinical forms revealed significantly decreased gene expression levels of EZH2, TLN1, and VAV1 in PBMC from RRMS, SPMS, and PPMS patients compared to HC (Fig. 1b). As depicted in Fig. 1c, mRNA expression levels for EZH2, TLN1, and VAV1 were not changed in RRMS patients at the time of acute exacerbations, and expression levels for these genes were similar between RRMS patients in clinical remission and RRMS patients during relapse.

EZH2 findings were validated in an independent cohort of 13 untreated multiple sclerosis patients and $12 \mathrm{HC}$, and mRNA expression levels for EZH2 were again found to be significantly decreased in PBMC from the multiple sclerosis group compared to the HC group ( $p=0.01$; Fig. $1 \mathrm{~d})$.

\section{Expression levels of miR-124 are increased in multiple sclerosis patients}

We next investigated the expression levels of miR-124 and miR-155, two microRNAs that are known on the one hand to target EZH2 $[8,9]$ and on the other hand to be involved in multiple sclerosis $[10,11]$. Following the determination of microRNA expression levels in 21 untreated multiple sclerosis patients and $18 \mathrm{HC}$, miR-124 expression was found to be significantly upregulated in PBMC from multiple sclerosis patients compared to controls $(p=0.03)$, whereas miR-155 expression levels were similar between patients and $\mathrm{HC}$ (Fig. 2). These results may suggest a potential and inverse relationship between EZH2 and miR-124 expression levels in multiple sclerosis patients.

\section{EZH2 expression is increased in treated multiple sclerosis patients}

As a next step, we investigated whether EZH2 and TLN1 expression was modulated by commonly used multiple sclerosis therapies. For this, mRNA expression levels for
EZH2 and TLN1 were determined in PBMC from 59 treated patients. Compared to untreated patients, EZH2 and TLN1 expression was significantly upregulated in PBMC by the effect of interferon-beta, Copaxone, and natalizumab treatments (Fig. 3). In contrast, whereas fingolimod significantly increased TLN1 expression, this treatment had no effect on EZH2 expression (Fig. 3). Overall, the increased EZH2 and TLN1 expression in PBMC from treated MS patients may indicate a reduced leukocyte trafficking into the CNS by the effect of treatment.

EZH2 is expressed by circulating CD4+ and CD8+ T cells with effector memory and central memory phenotypes respectively

In order to characterize the PBMC populations that express EZH2, immunophenotyping for EZH2 and flow cytometry analysis was performed in $\mathrm{T}$ cells (CD3+, CD4+, and CD8+), B cells, monocytes, and NK cells from 10 multiple sclerosis patients and $13 \mathrm{HC}$. EZH2 expression was restricted to $\mathrm{CD} 3+$ (both $\mathrm{CD} 4+$ and CD8+) T cells and CD56dim NK cells (Fig. 4a), whereas it was absent in B cells and monocytes. Similar to the mRNA expression findings observed in the whole PBMC population, the percentage of EZH2-positive cells in CD4+ and CD8+ T cells was significantly reduced in untreated multiple sclerosis patients compared to controls (Fig. 4a). Although treatment with natalizumab, which was selected as control therapy, increased EZH2 expression by T cells, differences did not reach statistical significance (Fig. 4a). In contrast, EZH2 expression by CD56dim NK cells was similar across the different groups (Fig. 4a).

Further, EZH2 immunophenotyping in naïve and different memory $\mathrm{T}$ cell populations revealed that $\mathrm{CD} 4+\mathrm{T}$ cells expressing EZH2 had a clear effector memory phenotype with low contribution of naïve T cells compared to CD4+ T cells negative for EZH2 expression (Fig. $4 \mathrm{~b}$ ). In contrast, CD8+ T cells expressing EZH2 exhibited a predominant central memory phenotype compared to EZH2-negative CD8+ T cells (Fig. 4c). A trend towards decreased expression of EZH2 $(p=0.07)$ was observed in terminally differentiated effector CD4+ T cells from untreated multiple sclerosis patients compared to $\mathrm{HC}$, and EZH2 expression was significantly upregulated in patients by the effect of natalizumab treatment $(p=0.03)$ (Fig. 4b). However, a similar pattern was also observed in terminally differentiated effector CD4+ T cells negative for EZH2 ( $p=0.05$ and $p=0.003$ in untreated patients versus controls and patients receiving treatment respectively) (Fig. 4b).

Finally, in order to evaluate the pathogenic potential of EZH2-positive cells, staining for proinflammatory cytokines such as TNF- $\alpha$, GM-CSF, and IL-17 was also included in CD4+ and CD8+ T cells from a subgroup of untreated $(N=4)$ and treated $(N=4)$ patients and $\mathrm{HC}$ 
A

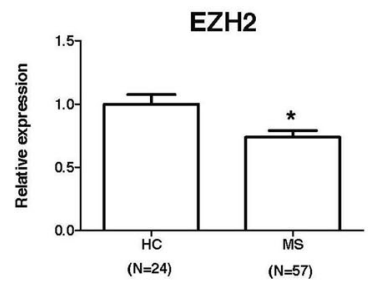

B

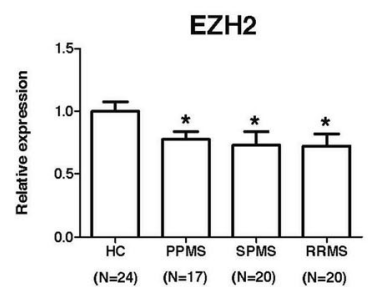

C

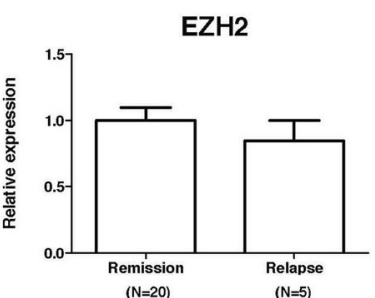

D EZH2 - Validation cohort

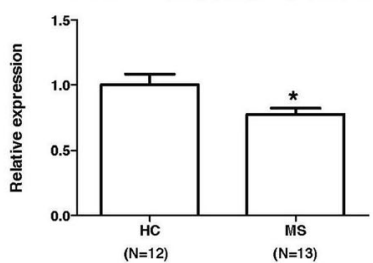

- Initial cohort -

TLN1

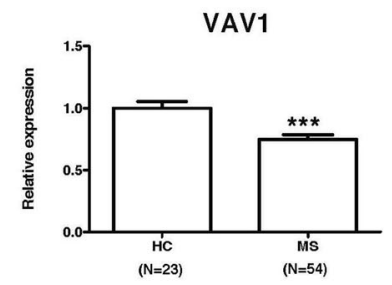

- Initial cohort -

TLN1
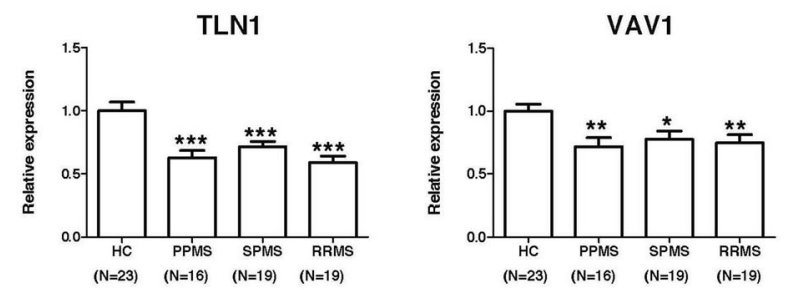

- Initial cohort -

TLN1
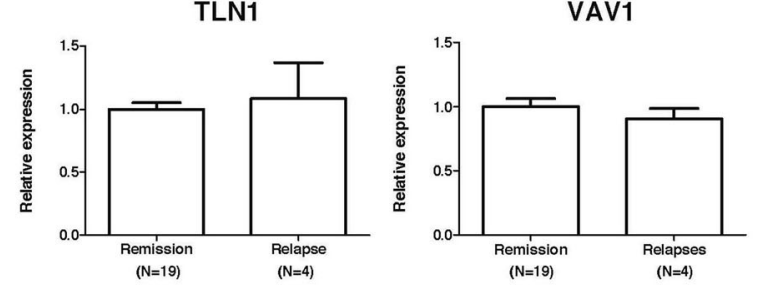

Fig. 1 Expression levels of EZH2 and EZH2-associated molecules in multiple sclerosis patients and controls. mRNA expression levels for EZH2, TLN1, and VAV1 were determined in PBMC from untreated multiple sclerosis patients and healthy controls by real-time PCR relative quantification, as described in the "Methods" section. Graphs showing expression levels for EZH2, TLN1, and VAV1 a-c in the initial discovery cohort and $\mathbf{d}$ in an independent cohort of patients and controls. Results are expressed as fold change (standard error of the mean) in gene expression in multiple sclerosis patients relative to controls and in patients in relapse relative to patients in remission. Statistics: unpaired Student's $t$ test. ${ }^{*} p$ values $<0.05 ;{ }^{*} p$ values $<0.01 ;{ }^{* * *} p$ values $<0.001$. HC healthy controls, MS whole group of multiple sclerosis patients, RRMS relapsing-remitting multiple sclerosis, SPMS secondary progressive multiple sclerosis, PPMS primary progressive multiple sclerosis, Remission RRMS patients in clinical remission, Relapse RRMS patients whose blood was collected at the time of an acute exacerbation, EZH2 enhancer of zeste 2 polycomb repressive complex 2 subunit, TLN1 talin 1, VAV1 vav guanine nucleotide exchange factor 1

$(N=7)$. EZH2-positive cells expressed TNF- $\alpha$ though were negative for GM-CSF and IL-17 expression. Interestingly, trends towards decreased percentage of TNF- $\alpha$-positive cells were observed in CD4+ and CD8+ T cells expressing EZH2 from untreated patients compared to $\mathrm{HC}(p=0.08$ and $p=0.07$ respectively), whereas no similar findings were seen in their EZH2-negative counterparts (Fig. 4d). Furthermore, natalizumab treatment was associated with significant increases in the percentage of TNF- $\alpha$-positive cells in
CD4+ and CD8+ T cells expressing EZH2 $(p=0.02$ and $p=0.04$ respectively), while treatment had no effect in the percentage of TNF- $\alpha$-positive cells by EZH2-negative CD4+ and CD8+ T cells (Fig. 4d).

Altogether, these data point to a different expression of EZH2 depending on the differentiation stages of the $\mathrm{CD} 4+$ and CD8+ T cells and suggest a common pathogenic potential of EZH2-positive cells in MS via TNF- $\alpha$ production. 


\section{Expression levels of microRNAs}

$\operatorname{miR}-124$

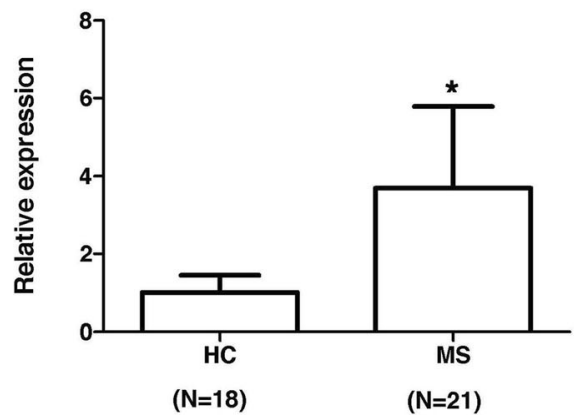

$\operatorname{miR}-155$

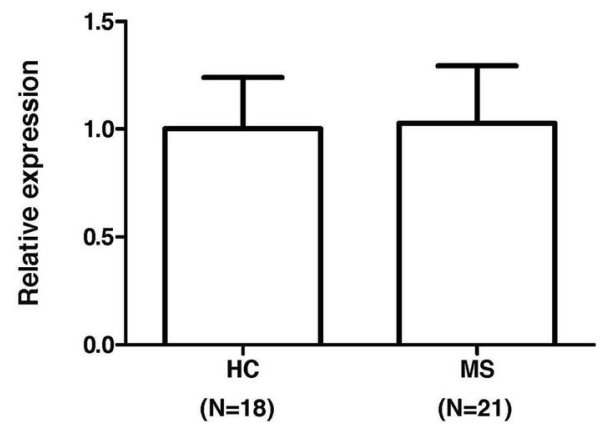

Fig. 2 Expression levels of miR-124 and miR-155 in multiple sclerosis patients and controls. Expression levels for miR-124 and miR-155 were determined in PBMC from untreated multiple sclerosis patients and controls by real-time PCR relative quantification. Results are expressed as fold change (standard error of the mean) in gene expression in patients relative to controls. Statistics: unpaired Student's $t$ test. ${ }^{*} p$ value $=0.03$. HC healthy controls, MS whole group of untreated multiple sclerosis patients, which included 15 RRMS and 6 SPMS patients

EZH2-positive T cells migrate to the CNS during EAE and multiple sclerosis

Based on the gene and protein expression findings, we hypothesized that the decrease of circulating EZH2-positive $\mathrm{T}$ cells in untreated multiple patients compared to controls was secondary to the migration of EZH2-positive T cells into the CNS. To evaluate this hypothesis, we first investigated EZH2 expression in the CNS of EAE mice and observed that EZH2 was expressed in spinal cord tissue during EAE, and EZH2 expression levels peaked at the inflammatory phase of the disease (day 16 postimmunization) (Fig. 5a). Interestingly, EZH2 followed a similar temporal pattern of CNS expression to $\mathrm{Cd} 3$ in EAE mice, and expression levels for these two genes correlated with each other (Spearman correlation coefficient $=$
0.63, $p=0.002$ ), suggesting a relationship between EZH2 expression and the inflammatory cell infiltrate during EAE (Fig. 5a). We next aimed to extrapolate these findings to patients with multiple sclerosis by determining EZH2 expression in CSF cells from three untreated patients. As shown in Fig. 5b, EZH2-positive $\mathrm{T}$ cells were enriched in the CSF compartment, and the percentage of CD3+ T cells expressing EZH2 was significantly increased in the CSF compared to peripheral blood $(p=0.007)$. In contrast, in CD56dim NK cells, a cell subset that also expressed EZH2 (Fig. 4a), the percentage of EZH2-positive cells did not differ between the CSF and blood compartments, indicating a preferential capacity for EZH2-positive T cells to migrate into the CNS. In this line, we finally investigated EZH2 expression in chronic active lesions from 10 multiple sclerosis

\section{Effect of treatment in EZH2 and TLN1 expression \\ EZH2 \\ TLN1}

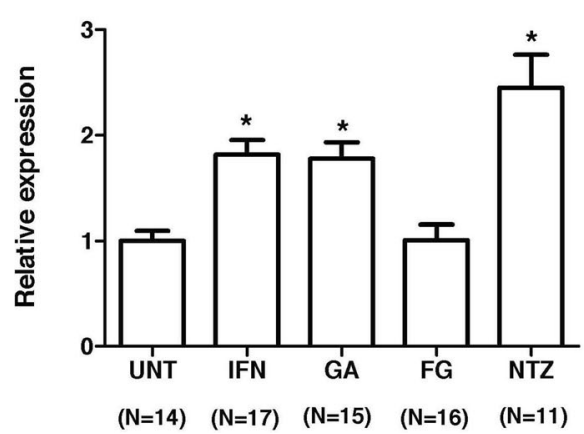

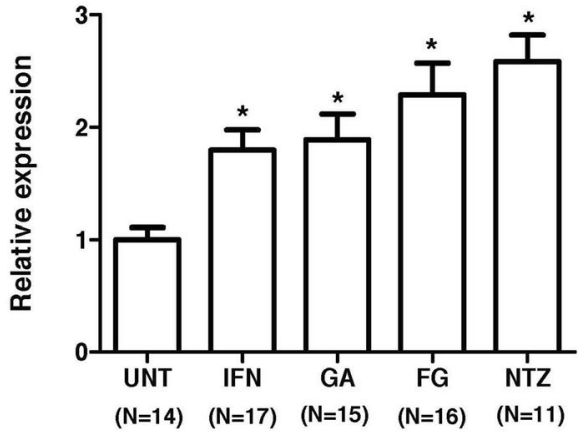

Fig. 3 Expression levels of EZH2 and TLN1 in treated multiple sclerosis patients. mRNA expression levels for EZH2 and TLN1 were determined by real-time PCR relative quantification in PBMC from untreated multiple sclerosis patients and patients receiving disease-modifying therapies for at least 1 year. Results are expressed as fold change (standard error of the mean) in gene expression in patients relative to controls. Statistics: MannWhitney $U$ test. ${ }^{*} p$ values $<0.001$. UNT untreated patients, IFN interferon-beta, GA glatiramer acetate, FG fingolimod, NTZ natalizumab, EZH2 enhancer of zeste 2 polycomb repressive complex 2 subunit, TLN1 talin 1 

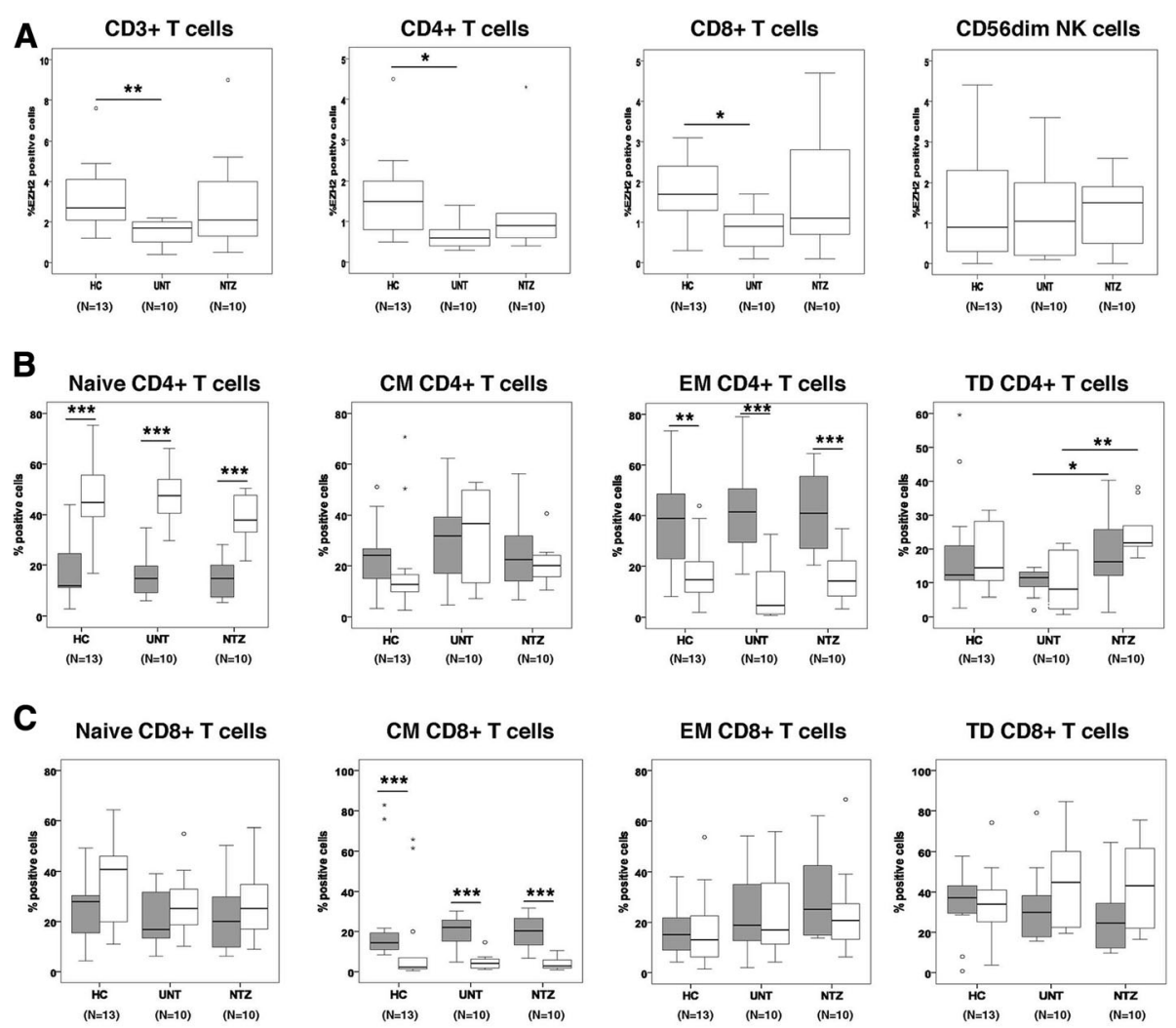

D

TNF- $\alpha$
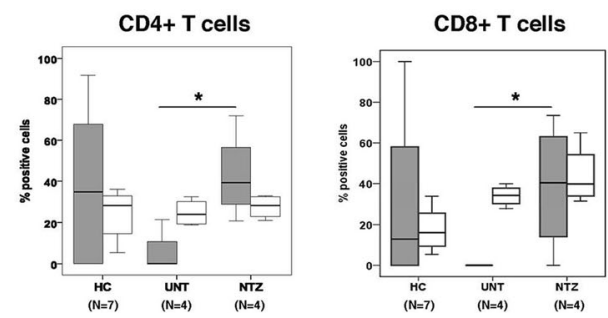

Fig. 4 EZH2 immunophenotyping in PBMC from multiple sclerosis patients and controls. a Graphs showing the percentage of EZH2-positive cells in T cells and CD56dim NK cells from healthy controls (HC; $n=13)$, untreated multiple sclerosis patients (UNT; $n=10)$, and the same cohort of patients after 1 year of natalizumab treatment $(N T Z ; n=10)$. b, c Graphs showing EZH2 expression in different CD4+ and CD8+ T cell subsets (HC, $n=13 ; \mathrm{UNT}, n=10 ; \mathrm{NTZ}, n=10)$. Naïve T cells were defined as CD45RO-/CCR7+. Central memory (CM) T cells were defined as CD45RO+/CCR7+. Effector memory (EM) T cells were defined as CD45RO+/CCR7-. Terminally differentiated (TD) effector T cells were defined as CD45RO-/CCR7+. Gray boxes, EZH2-positive cells; open boxes, EZH2-negative cells. $\mathbf{d}$ Graphs showing the percentage of tumor necrosis factor (TNF)-a-positive cells in CD4+ and CD8+ T cells expressing EZH2 (HC, $n=7 ; \mathrm{UNT}, n=4 ; \mathrm{NTZ}, n=4)$. Gray boxes, EZH2-positive cells; open boxes, EZH2-negative cells. Statistics: $\mathbf{a}, \mathbf{b}$ unpaired Student's $t$ test; $\mathbf{c}$, d Mann-Whitney $U$ test. ${ }^{*} p$ values $<0.05 ;{ }^{* *} p$ values $<0.01 ;{ }^{* * *} p$ values $<0.001$. EZH2 enhancer of zeste 2 polycomb repressive complex 2 subunit

patients and observed that $8.5 \%$ (mean percentage) and $10.9 \%$ of the total CD4+ and CD8+ T cells were expressing EZH2 respectively (Fig. 5c). Occasionally, EZH2 expression was also observed in the nuclei of few glial cells, and brain tissues from non-neurological controls were negative for EZH2 expression (data not shown).

\section{EZH2 blocking downregulates T cell adhesion molecules} As a last step, we incubated in vitro PBMC from seven untreated multiple sclerosis patients with an EZH2 inhibitor in order to investigate the genes modulated by EZH2 and aiming to better understand the role of EZH2 in disease pathophysiology. A total of 6763 genes were significantly up- or downregulated by the effect of the EZH2 inhibitor (with $p$ values $<0.05$; data not shown). Interestingly, among the top $1 \%$ of differentially expressed genes between the untreated and treated conditions, we identified key $\mathrm{T}$ cell adhesion molecules that were strikingly downregulated by the EZH2 inhibitor such as selectin L (SELL, also known as CD62L; adjusted $p$ value versus the 


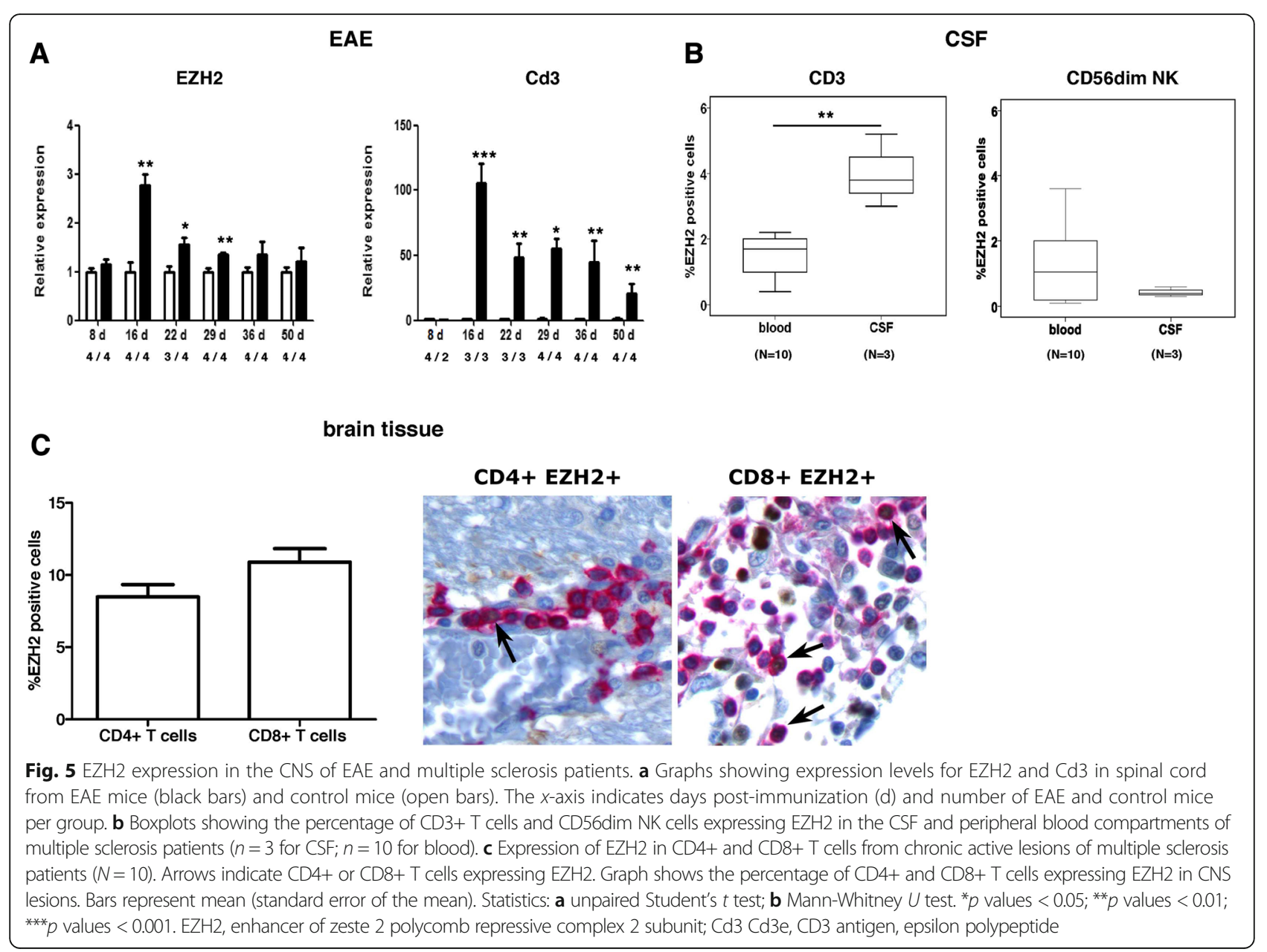

untreated condition $\left.=6.4 \times 10^{-20}\right)$, integrin subunit alpha 4 (ITGA4, also known as CD49D; $p=3.6 \times 10^{-16}$ ), integrin subunit alpha L (ITGAL, also known as CD11A; $p=1.1 \times$ $\left.10^{-13}\right)$, and platelet and endothelial cell adhesion molecule 1 (PECAM1; $p=5.2 \times 10^{-14}$ ) (Fig. 6). These data support a role of EZH2 in the adhesion of circulating T cells.

\section{Discussion}

Extensive literature exists about the role of EZH2 as histone methyltransferase and, particularly, about EZH2 involvement in a wide range of malignant tumors $[8,12,13]$ due to its function as epigenetic silencer [14]. The identification of a cytosolic methyltransferase complex containing EZH2 suggested that, in addition to its nuclear role, EZH2 could also be involved in other important cellular processes such as cell adhesion and migration [2]. In an attempt to characterize more in depth the cytosolic role of EZH2, Gunawan et al. [2] recently reported that EZH2 was critical for regulating leukocyte migration to sites of inflammation in EAE mice, findings that opened a potential and attractive link between EZH2 and autoimmune disorders such as multiple sclerosis in which cell adhesion and migration are critical pathogenic mechanisms [15]. Despite this initial publication in the animal model of multiple sclerosis [3], to date, there are no studies of EZH2 in patients with multiple sclerosis. Aiming to explore the role of EZH2 in multiple sclerosis, we first determined mRNA expression levels in PBMC from untreated patients and healthy individuals and observed that EZH2, together with molecules reported to be associated with cytosolic EZH2 such as TLN1 and VAV1 [3], were all downregulated in multiple sclerosis patients regardless of whether they were having relapse-onset or progressive clinical forms, or whether they were in clinical remission or in acute relapse. The decreased expression of EZH2 in PBMC from patients with multiple sclerosis was replicated in an independent validation cohort of patients and controls. These initial observations at the gene expression level suggested an involvement of EZH2 in the disease, which was explored in additional experiments.

We first explored microRNAs, which are known to exert regulatory functions at the posttranscriptional level via binding to the $3^{\prime}$ untranslated region of target mRNAs 

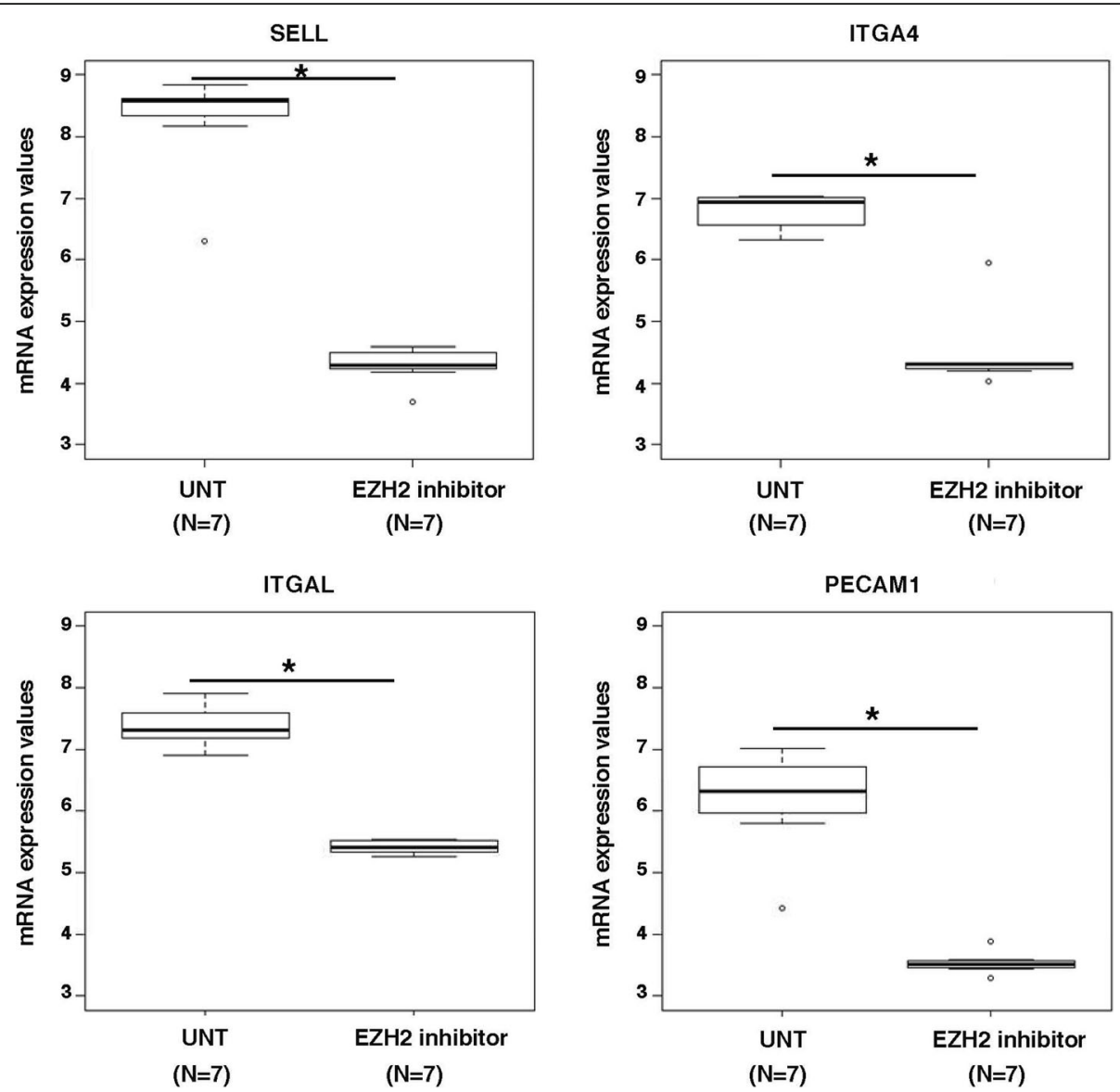

Fig. 6 Expression of cell adhesion molecules in PBMC following EZH2 blocking. Boxplots showing expression levels obtained with microarrays for adhesion molecules before and after incubation of PBMC from multiple sclerosis patients with an EZH2 inhibitor for $24 \mathrm{~h}$. The asterisk symbol refers to adjusted $p$ values $<0.001$. UNT untreated condition, SELL selectin L, ITGA4 integrin subunit alpha 4, ITGAL integrin subunit alpha L, PECAM1 platelet and endothelial cell adhesion molecule 1

[16]. In the search for potential microRNAs that on the one hand regulated EZH2 expression and on the other hand were involved in multiple sclerosis, the microRNAs miR-124 and miR-155 emerged as attractive candidates $[8,9]$. In this context, expression levels for miR-124 were found increased in demyelinated hippocampi from postmortem brains of multiple sclerosis patients [10]. miR-155 expression was upregulated in peripheral blood monocytes and active lesions from patients [11]. In our study, when expression levels for these two microRNAs were determined in PBMC from a subgroup of untreated multiple sclerosis patients and controls, miR-124 but not miR-155 was significantly upregulated in patients, suggesting a potential functional relationship between decreased EZH2 mRNA expression levels and miR-124 upregulation in patients with multiple sclerosis. These findings warrant future studies to investigate the cell types that contribute to miR-124 upregulation.

In order to explore whether the expression of EZH2 and associated molecules was modulated by commonly used disease-modifying therapies in patients with multiple sclerosis, a cohort of patients treated with interferon-beta, glatiramer acetate, fingolimod, and natalizumab was also included in the study. Treatment with interferon-beta, glatiramer acetate, and natalizumab was associated with increased expression levels of EZH2 and TLN1. By contrast, fingolimod treatment was only associated with upregulated expression of TLN1. Although based on a small number of samples and in spite of the descriptive nature of the experiments, it is tempting to speculate that this finding may be due to the different mechanisms by which these drugs regulate leukocyte migration to the CNS, being the mechanism of action of fingolimod not exerted in peripheral blood but in the lymph nodes where it retains naive and central memory lymphocytes [17-20]. In view of these data, we hypothesize that the reduction in EZH2 expression observed in untreated patients with subsequent upregulation after treatment may indicate a migration capacity of PBMC expressing EZH2 to the CNS that is inhibited by the effect of treatment. These findings also open a new research 
avenue to investigate whether the increase in EZH2 expression observed after treatment is associated with the response to therapies and hence may differ between responders and non-responders to each particular therapeutic strategy.

Immunophenotyping of the major $\mathrm{PBMC}$ populations revealed restricted EZH2 expression in CD4+ and CD8+ $\mathrm{T}$ cells as well as CD56dim NK cells. Similar to the gene expression findings, the percentage of EZH2-positive $\mathrm{T}$ cells was reduced in untreated multiple sclerosis patients compared to controls, pointing to a role of EZH2 in this particular cell subset rather than in CD56dim NK cells, which showed similar percentages of EZH2-positive cells in patients and controls. It is worth highlighting that except for one patient and one control, the immunophenotyping cohort included new individuals, and hence, the decrease of EZH2-positive T cells observed in patients can also be considered as a new validation of the EZH2 expression findings at the protein level. Natalizumab, which was selected as control therapy because of its known effects reducing $\mathrm{T}$ cell trafficking into the CNS, increased the percentage of EZH2-positive T cells, but, contrary to gene expression results, differences did not reach statistical significance. Interestingly, further $\mathrm{T}$ cell immunophenotyping showed heterogeneity in the $\mathrm{T}$ cell subsets positive for EZH2 expression. In this context, most CD4+ T cells expressing EZH2 were effector memory $\mathrm{T}$ cells, a population that per se has the capacity to migrate to non-lymphoid tissues including the CNS [21]. In contrast, $\mathrm{CD} 8+\mathrm{T}$ cells positive for EZH2 had a predominant central memory phenotype, suggesting that this particular subset can migrate to the CNS in MS and may later differentiate into effector memory populations in the sites of inflammation. Of note, EZH2-positive cells both in CD4+ and CD8+ T cells may have pathogenic potential through the secretion of TNF- $\alpha$, a pro-inflammatory cytokine involved in the pathogenesis of multiple sclerosis [22]. Although caution should be taken when considering these data owing to the small sample size and high variability, these findings altogether warrant additional studies to deepen into the pathogenic capacity of $\mathrm{T}$ cells expressing EZH2 and explore whether EZH2 may become a therapeutic target in MS patients to reduce disease activity.

The potential for EZH2-positive T cells to migrate to the CNS was first suggested in the EAE study, which showed EZH2 expression in spinal cord tissue from EAE mice following a similar pattern to $\mathrm{Cd} 3$ expression over time. Confirmation of the migratory capacity of EZH2-positive $\mathrm{T}$ cells was provided by their detection in the CSF and brain lesions from multiple sclerosis patients. Noteworthy, the enrichment for CD4+ and CD8+ T cells expressing $\mathrm{EZH} 2$ in the CSF and chronic active lesions compared to the blood compartment suggested that the decrease of EZH2-positive $\mathrm{T}$ cells observed in untreated multiple sclerosis patients compared to healthy individuals was secondary to their migration into the CNS. This notion was further supported by the observation that other EZH2-expressing blood cell populations such as CD56dim NK cells, which did not depict differences between patients and controls in blood, were not enriched in the CSF. Although EZH2 expression in brain lesions from multiple sclerosis patients seemed restricted to CD4+ and CD8+ T cells, additional sources of EZH2 expression within the CNS cannot be totally ruled out, as evidenced by EZH2 immunohistochemistry. In contrast, EZH2 expression was not observed in brain tissue from non-neurological controls, a finding that confers specificity for EZH2 expression in the CNS of multiple sclerosis patients.

\section{Conclusions}

Finally, the implication of EZH2 in T cell adhesion, an important step for cell migration, was supported by the microarray findings conducted after EZH2 blocking with a histone deacetylase inhibitor that regulates EZH2 expression [23], which showed striking downregulation of cell adhesion molecules expressed in T cells such as SELL [24], ITGA4 [25], ITGAL [26], and PECAM1 [27]. The aggregate results from the study suggest a role for EZH2 in the migration of $\mathrm{T}$ cells into the CNS in patients with multiple sclerosis and also suggest a potential pathogenic capacity of EZH2-positive T cells that will need to be explored more in depth in future studies.

\section{Additional file}

Additional file 1: Table S1. Demographic and clinical characteristics of the multiple sclerosis patients and $\mathrm{HC}$ included for the determination of microRNA expression levels. (DOC $36 \mathrm{~kb}$ )

\section{Abbreviations}

EAE: Experimental autoimmune encephalomyelitis; EZH2: Enhancer of zeste 2 polycomb repressive complex 2 subunit; GM-CSF: Granulocyte/macrophage colony-stimulating factor; H3K27: Histone $\mathrm{H} 3$ at lysine 27; HC: Healthy controls; ITGA4: Integrin subunit alpha 4; ITGAL: Integrin subunit alpha L; KB: Klüver-Barrera; mRNA: Messenger RNA; MS: Multiple sclerosis; PBMC: Peripheral blood mononuclear cells; PECAM1: Platelet and endothelial cell adhesion molecule 1; PMA: Phorbol 12-myristate 13-acetate; PPMS: Patients with primary progressive multiple sclerosis; RRMS: Relapsing-remitting multiple sclerosis; SAHA: Suberoylanilide hydroxamic acid; SELL: Selectin L;

TLN1: Talin 1; TNF: Tumor necrosis factor

\section{Acknowledgements}

The authors would like to thank the nurses, laboratory technicians, and patients for their participation in sample collection.

\section{Funding}

The authors thank the "Red Española de Esclerosis Múltiple (REEM)" sponsored by the FEDER-FIS and the "Ajuts per donar Suport als Grups de Recerca de Catalunya," sponsored by the "Agència de Gestió d'Ajuts Universitaris i de Recerca" (AGAUR), Generalitat de Catalunya, Spain.

Availability of data and materials

The dataset used or analyzed for the current study is available from the corresponding author on reasonable request. 


\section{Authors' contributions}

$\mathrm{SM}, \mathrm{LMV}, \mathrm{XM}$, and $\mathrm{MC}$ contributed to the conception and study design. CC, LM, MCu SMe, NF, JR, JC, JCA-C, and AS contributed to the data acquisition and analysis. SM, LMV, and MC contributed to the drafting of the manuscript and figures. All authors read and approved the final manuscript.

\section{Ethics approval and consent to participate}

Experiments were done according to the EU regulations and approved by our institutional Ethics Committee on Animal Experimentation. The study was approved by the local Ethics Committee, and participants gave written informed consent.

\section{Consent for publication}

Not applicable.

\section{Competing interests}

The authors declare that they have no competing interests.

\section{Publisher's Note}

Springer Nature remains neutral with regard to jurisdictional claims in published maps and institutional affiliations.

\section{Author details}

'Servei de Neurologia-Neuroimmunologia, Centre d'Esclerosi Múltiple de Catalunya (Cemcat), Institut de Recerca Vall d'Hebron (VHIR), Hospital Universitari Vall d'Hebron, Universitat Autònoma de Barcelona, Barcelona, Spain. ${ }^{2}$ Departments of Neurology and Immunology, Hospital Universitario Ramón y Cajal, Instituto Ramón y Cajal de Investigacion Sanitaria, Madrid, Spain. ${ }^{3}$ Departament d'Estadística, Facultat de Biologia, Universitat de Barcelona, Barcelona, Spain. ${ }^{4}$ Unitat d'Estadística i Bioinformàtica, Institut de Recerca, HUVH, Barcelona, Spain. ${ }^{5}$ Genetics, Microbiology and Statistics Department, Universitat de Barcelona, Barcelona, Spain.

\section{Received: 25 June 2018 Accepted: 16 October 2018}

Published online: 26 October 2018

\section{References}

1. Cao R, Wang L, Wang H, Xia L, Erdjument-Bromage H, Tempst P, et al. Role of histone $\mathrm{H3}$ lysine 27 methylation in polycomb-group silencing. Science. 2002;298:1039-43.

2. Su IH, Dobenecker MW, Dickinson E, Oser M, Basavaraj A, Marqueron R, et al. Polycomb group protein ezh2 controls actin polymerization and cell signaling. Cell. 2005;121:425-36.

3. Gunawan M, Venkatesan N, Loh JT, Wong JF, Berger H, Neo WH, et al. The methyltransferase Ezh2 controls cell adhesion and migration through direct methylation of the extranuclear regulatory protein talin. Nat Immunol. 2015. 2015;16:505-16.

4. Livak KJ, Schmittgen TD. Analysis of relative gene expression data using real-time quantitative PCR and the 2(-Delta Delta C(T)). Methods. 2001;25: 402-8.

5. Irizarry RA, Hobbs B, Collin F, Beazer-Barclay YD, Antonellis K, Scherf U, et al. Exploration, normalization, and summaries of high density oligonucleotide array probe level data. Biostatistics. 2003;4:249-64.

6. Smyth GK. Linear models and empirical Bayes methods for assessing differential expression in microarray experiments. Stat Appl Genet Mol Biol. 2004;3:Article3.

7. Benjamin Y, Hochberg Y. Controlling the false discovery rate: a practical and powerful approach to multiple testing. J Roy Stat Soc B. 1995;57:289-30.

8. Zheng F, Liao YJ, Cai MY, Liu YH, Liu TH, et al. The putative tumour suppressor microRNA-124 modulates hepatocellular carcinoma cell aggressiveness by repressing ROCK2 and EZH2. Gut. 2012;61:278-89.

9. Mei S, Liu Y, Bao Y, Zhang Y, Min S, Liu Y, et al. Dendritic cell-associated miRNAs are modulated via chromatin remodeling in response to different environments. PLoS One. 2014;9:e90231.

10. Dutta R, Chomyk AM, Chang A, Ribaudo MV, Deckard SA, Doud MK, et al. Hippocampal demyelination and memory dysfunction are associated with increased levels of the neuronal microRNA miR-124 and reduced AMPA receptors. Ann Neurol. 2013;73:637-45.

11. Moore CS, Rao VT, Durafourt BA, Bedell BJ, Ludwin SK, Bar-Or A, et al. miR155 as a multiple sclerosis-relevant regulator of myeloid cell polarization. Ann Neurol. 2013;74:709-20.
12. Lee SR, Roh YG, Kim SK, Lee JS, Seol SY, Lee HH, et al. Activation of EZH2 and SUZ12 regulated by E2F1 predicts the disease progression and aggressive characteristics of bladder cancer. Clin Cancer Res. 2015;21:5391-403.

13. Zingg D, Debbache J, Schaefer SM, Tuncer E, Frommel SC, Cheng P, et al. The epigenetic modifier EZH2 controls melanoma growth and metastasis through silencing of distinct tumour suppressors. Nat Commun. 2015;6:6051.

14. Sun S, Yu F, Zhang L, Zhou X. EZH2, an on-off valve in signal network of tumor cells. Cell Signal. 2016;28:481-7.

15. Sospedra M, Martin R. Immunology of multiple sclerosis. Annu Rev Immunol. 2015;23:683-747.

16. Macfarlane LA, Murphy PR. MicroRNA: biogenesis, function and role in cancer. Curr Genom. 2010;11:537-61.

17. Niino M, Bodner C, Simard ML, Alatab S, Gano D, Kim HJ, et al. Natalizumab effects on immune cell responses in multiple sclerosis. Ann Neurol. 2006;59: $748-54$.

18. Dhib-Jalbut S, Marks S. Interferon-beta mechanisms of action in multiple sclerosis. Neurology. 2010;74:S17-24.

19. Griffith JW, Luster AD. Targeting cells in motion: migrating toward improved therapies. Eur J Immunol. 2013:43:1430-5.

20. Sellner J, Koczi W, Harrer A, Oppermann K, Obregon-Castrillo E, Pilz G, et al. Glatiramer acetate attenuates the pro-migratory profile of adhesion molecules on various immune cell subsets in multiple sclerosis. Clin Exp Immunol. 2013;173:381-9.

21. Mueller SN, Gebhardt T, Carbone FR, Heath WR. Memory T cell subsets, migration patterns, and tissue residence. Annu Rev Immunol. 2013;31:137-61.

22. Caminero A, Comabella M, Montalban X. Tumor necrosis factor alpha (TNF-a), anti-TNF- $a$ and demyelination revisited: an ongoing story. J Neuroimmunol. 2011;234:1-6.

23. Yamaguchi J, Sasaki M, Sato Y, Itatsu K, Harada K, Zen Y, et al. Histone deacetylase inhibitor (SAHA) and repression of $\mathrm{EZH} 2$ synergistically inhibit proliferation of gallbladder carcinoma. Cancer Sci. 2010;101:355-62.

24. Wedepohl S, Beceren-Braun F, Riese S, Buscher K, Enders S, Bernhard G, et al. L-selectin--a dynamic regulator of leukocyte migration. Eur J Cell Biol. 2012;91:257-64.

25. Cobo-Calvo Á, Figueras A, Bau L, Matas E, Mañé Martínez MA, León I, et al. Leukocyte adhesion molecule dynamics after natalizumab withdrawal in multiple sclerosis. Clin Immunol. 2016:171:18-24.

26. Jilek S, Mathias A, Canales M, Lysandropoulos A, Pantaleo G, Schluep M, et al. Natalizumab treatment alters the expression of T-cell trafficking marker LFA-1 a-chain (CD11a) in MS patients. Mult Scler. 2014;20:837-42.

27. Qing Z, Sandor M, Radvany Z, Sewell D, Falus A, Potthoff D, et al. Inhibition of antigen-specific $T$ cell trafficking into the central nervous system via blocking PECAM1/CD31 molecule. J Neuropathol Exp Neurol. 2001;60:798-807.

Ready to submit your research? Choose BMC and benefit from:

- fast, convenient online submission

- thorough peer review by experienced researchers in your field

- rapid publication on acceptance

- support for research data, including large and complex data types

- gold Open Access which fosters wider collaboration and increased citations

- maximum visibility for your research: over $100 \mathrm{M}$ website views per year

At BMC, research is always in progress.

Learn more biomedcentral.com/submission 